\title{
How a Kerr-Newman Black Hole Leads to Criteria about If Gravity Is Quantum Due to Questions on If $(\Delta t)^{5}+A_{1} \cdot(\Delta t)^{2}+A_{2}=0$ Is Solvable
}

\author{
Andrew Walcott Beckwith \\ Physics Department, College of Physics, Huxi Campus, Chongqing University, Chongqing, China \\ Email: Rwill9955b@gmail.com, abeckwith@uh.edu
}

How to cite this paper: Beckwith, A.W. (2019) How a Kerr-Newman Black Hole Leads to Criteria about If Gravity Is Quantum Due to Questions on If

$(\Delta t)^{5}+A_{1} \cdot(\Delta t)^{2}+A_{2}=0$ Is Solvable. Journal of High Energy Physics, Gravitation and Cosmology, 5, 35-40.

https://doi.org/10.4236/jhepgc.2019.51002

Received: October 12, 2018

Accepted: December 3, 2018

Published: December 6, 2018

Copyright $\odot 2019$ by author and Scientific Research Publishing Inc. This work is licensed under the Creative Commons Attribution International License (CC BY 4.0).

http://creativecommons.org/licenses/by/4.0/

\begin{abstract}
Note, in a prior paper, we ascertained physics thought experiment configuration for a black hole, which may exist say at least up to $10^{-1}$ seconds. Our idea was to experimentally provide a test bed as to early universe gravitational theories. In doing so, we as follow up to that black hole paper come up with a criteria as to Quintic polynomial with regards to $\Delta t$ which is the interval of time for which we can measure (down to Planck time) the production of Gravitational waves and gravitons, from an induced Kerr-Newman black hole. In doing so we access what is given in an AdS/CFT rendition of black hole entropy written by Pires which gives an input strategy as to how to relate $\Delta t$ to a $(\Delta t)^{5}+A_{1} \cdot(\Delta t)^{2}+A_{2}=0$ Quintic polynomial which has only a few combinations which may be exactly solvable. We find that $A_{2}$ has a number, $\mathrm{n}$ of presumed produced gravitons, in the time interval $\Delta t$ and that both $A_{1}$ and $A_{2}$ have an Ergosphere area, due to the induced Kerr-Newman black hole. Finally, we extract information via the use of the Uncertainty Principle, as to $\Delta E \Delta t \geq \hbar$ with $\Delta E \propto E_{0} \equiv m c^{2}$, so if we have a mass $\mathrm{m}$, we will be able to extract $\Delta t$. This due to very complete arguments as to Kerr-Newman black holes, which when we have entropy, due to the Infinite quantum statistics argument given by $\mathrm{Ng}$, leads to a counting algorithm, of $\mathrm{n}$ gravitons, which is proportional to entropy during $\Delta t$ which is then leading directly to fixing $\Delta t$ directly via us of $(\Delta t)^{5}+A_{1} \cdot(\Delta t)^{2}+A_{2}=0$, with the Quintic evaluated according to Blair K. Spearman and Kenneth S. Williams, in the Rocky mountain journal of mathematics, as of 1996. i.e. if this polynomial, as by our described Quintic polynomial, in $\Delta t,(\Delta t)^{5}+A_{1} \cdot(\Delta t)^{2}+A_{2}=0$ is exactly
\end{abstract}


solvable, then our Kerr Newman black hole is leading to quantum gravity. Otherwise, gravity in its foundations with respect to the Kerr Newman blackhole is classical to semi classical. In its characterization of gravity. Note that specifically, we state that this paper is modeling the creation of an actual Kerr Newman black hole via laser physics, or possibly by other means and that our determination of $\Delta t$ as being solved, exactly by

$(\Delta t)^{5}+A_{1} \cdot(\Delta t)^{2}+A_{2}=0$ is our way of determining if the Kerr Newman black hole leads to quantum gravity.

\section{Keywords}

Kerr Newman Black Hole, High-Frequency Gravitational Waves (HGW), Solvable Quintic Equations

\section{Introduction}

With regards to this problem, it is useful to make reference to [1], as its review of the fact that a general solution to Quintic $5^{\text {th }}$ order polynomials does not exist. What we are doing is accessing instead results from Galois theory, as to Quintics, [2] [3] [4]. For the case of the Quintics polynomial we are going to derive, namely $(\Delta t)^{5}+A_{1} \cdot(\Delta t)^{2}+A_{2}=0$ we refer the reader to the following document, [5] which has a restricted number of Quintic polynomials which are solvable according to the diagnostics given in that article. But in any case the Quintic polynomial with regards to $(\Delta t)^{5}+A_{1} \cdot(\Delta t)^{2}+A_{2}=0$ will be derived as an extension of the discussion given by the author in [6] as to an induced KerrNewman black hole. As to future developments, this has a tie into [7] as to the Riemann Zeta function and Riemann $\mathrm{P}$ function, which may be tied into this documents developments in a later discussion. Whereas, we will confine ourselves to utilizing an interval of time, $\Delta t$ as being affected by the production of gravitons, of a numerical counting value of $n$, whereas this is then linked to entropy, by the infinite quantum statistics argument of $\mathrm{Ng}[8]$, so that. $\Delta t$ has a functional dependence upon the number of gravitons, n, in msasurements, as alluded to in [6].

In a nutshell, we will be formally deriving $(\Delta t)^{5}+A_{1} \cdot(\Delta t)^{2}+A_{2}=0$ in our next section and from there ascertaining if the polynomial so derived, is explainable in terms of [5], in terms of exactly solvable solutions for $\Delta t$. For the sake of referencing the development of this article, we have as our motivating hypothesis, that if $(\Delta t)^{5}+A_{1} \cdot(\Delta t)^{2}+A_{2}=0$ is a polynomial in a form given in [5] that indeed, since $n$ will be in terms of a graviton count from a black hole given in [6], that then we have a necessary condition for quantum gravity, at least in the framework of aligning $(\Delta t)^{5}+A_{1} \cdot(\Delta t)^{2}+A_{2}=0$ in terms of the polynomials given in [5] which are allegedly exactly solvable. If $(\Delta t)^{5}+A_{1} \cdot(\Delta t)^{2}+A_{2}=0$ does not meet the conditions given in [5], then we say that the criteria for exact sol- 
vability of an expression for $\Delta t$ have not been met, and that indeed, then we have at best a semi classical treatment of gravity for reason which we will discuss at the end of our manuscript.

Finally, the reference [9] by C. A. Pickett and J. D. Zunda gives an area calculation which neatly fits into [10] and [11], whereas there is in [10] a precise calculation of entropy which also has an area to volume identification for black holes and entropy calculations. We close after all of this in stating that the energy, will be part of $\Delta E$, as in the usual Heisenberg Uncertainty relationships, $\Delta E \Delta t \geq \hbar$, whereas we take the minimum condition of uncertainty by writing $\Delta E \Delta t \equiv \hbar$ [12], and [13] confirms that indeed we have that use of minimum uncertainty in terms of data analysis has a long history if done correctly. Keep in mind that we do an abbreviation of

$$
\Delta E \equiv m c^{2}=\hbar / \Delta t \Rightarrow m=\hbar / c \Delta t
$$

This will allow us to obtain, in entropy, as from [10] and [11] a polynomial which we identify as $(\Delta t)^{5}+A_{1} \cdot(\Delta t)^{2}+A_{2}=0$. The exact solution of this analysis, in terms of [3] will then form the basis of our analysis of if we have classical gravity, or quantum gravity, in terms of necessary conditions. If Equation (1) and $(\Delta t)^{5}+A_{1} \cdot(\Delta t)^{2}+A_{2}=0$ is not exactly solvable, in terms of [5] we will the assert that this means gravity, in the case of the derived expression for Kerr Newman black holes, is semi classical.

\section{Derivation of the Polynomial $(\Delta t)^{5}+A_{1} \cdot(\Delta t)^{2}+A_{2}=0$}

We begin by looking at [10] and [11] for which we have that in terms of an AdS/CFT representation of entropy that we have, especially if we use [9] for Area, and $S$ proportional to $n$ for graviton count related to Entropy, as by [8], then

$$
\begin{aligned}
& A_{\text {area }}=16 \pi m^{2}+\frac{4 \pi}{3} \cdot\left(\frac{J}{m}\right)^{3}=16 \pi \cdot(\hbar / c \Delta t)^{2}+\frac{4 \pi}{3} \cdot\left(\frac{J c \cdot \Delta t}{\hbar}\right)^{3} \\
& \mathrm{~d} S^{2}=\frac{L^{2}}{r^{2}} \cdot\left(-\left(1-\left(\frac{r}{r_{+}}\right)^{2}\right) \mathrm{d} t^{2}+\frac{\mathrm{d} r^{2}}{\left(1-\left(\frac{r}{r_{+}}\right)^{2}\right)}+\mathrm{d} x^{i} \mathrm{~d} x^{j}\right) \\
& S_{\text {entropy }}=\frac{L^{d-1}}{4 G_{N}} \cdot\left(\frac{r}{r_{+}}\right)^{d-1} \cdot\left(16 \pi \cdot(\Delta E)^{2}+\frac{4 \pi}{3} \cdot\left(\frac{J c^{2}}{\Delta E}\right)^{3}\right) \cdot\left(\frac{4 \pi T_{\text {temp }}}{d}\right)^{d-1} \propto n_{\text {graviton-count }}
\end{aligned}
$$

We then have the following representation for a polynomial in $\Delta t$, namely if we have conflating of the material in Equation (2) as far as a quantic treatment of delta $t$, as by [5] we have that

$$
\frac{L^{d-1}}{4 G_{N}} \cdot\left(\frac{r}{r_{+}}\right)^{d-1} \cdot\left(16 \pi \cdot(\hbar / \Delta t)^{2}+\frac{4 \pi}{3} \cdot\left(\frac{J c^{2}}{(\hbar / \Delta t)}\right)^{3}\right) \cdot\left(\frac{4 \pi T_{\text {temp }}}{d}\right)^{d-1} \propto n_{\text {graviton-count }}
$$

We will then, describe how to obtain from Equation (3) 
$(\Delta t)^{5}+A_{1} \cdot(\Delta t)^{2}+A_{2}=0$

\section{Obtaining $(\Delta t)^{5}+A_{1} \cdot(\Delta t)^{2}+A_{2}=0$ from Equation (3)}

In order to obtain this, we make the following substitutions below, and we will state specifically that in order to have a negative temperature in order to obtain the conditions as given in [5] for a Quintic polynomial which is solvable in the sense of what that article [5] is saying. We will later on describe this in detail. But below we put in the substation needed so we can obtain the polynomial in delta $t$, which we will then subsequently modify.

$$
\begin{aligned}
& \frac{L^{d-1}}{4 G_{N}} \cdot\left(\frac{r}{r_{+}}\right)^{d-1} \cdot\left(16 \pi \cdot(\hbar / \Delta t)^{2}+\frac{4 \pi}{3} \cdot\left(\frac{J c^{2}}{(\hbar / \Delta t)}\right)^{3}\right) \cdot\left(\frac{4 \pi T_{\text {temp }}}{d}\right)^{d-1} \propto n_{\text {graviton-count }} \\
& \Rightarrow\left(16 \pi \cdot(\hbar / \Delta t)^{2}+\frac{4 \pi}{3} \cdot\left(\frac{J c^{2}}{(\hbar / \Delta t)}\right)^{3}\right) \propto \frac{n_{\text {graviton-count }}}{\left(\frac{4 \pi T_{\text {temp }}}{d}\right)^{d-1}} \\
& \Rightarrow\left(16 \pi \cdot(\hbar)^{2}+(\Delta t)^{5} \cdot \frac{4 \pi}{3} \cdot\left(\frac{J c^{2}}{\hbar}\right)^{3}\right)-\frac{n_{\text {graviton-count }}}{\left(\frac{4 \pi T_{\text {temp }}}{d}\right)^{d-1}}(\Delta t)^{2} \equiv 0 \\
& \Rightarrow(\Delta t)^{5}-\frac{n_{\text {graviton-count }}}{\left(\frac{4 \pi T_{\text {temp }}}{d}\right)^{d-1} \cdot \frac{4 \pi}{3} \cdot\left(\frac{J c^{2}}{\hbar}\right)^{3}}(\Delta t)^{2}+\frac{16 \pi \cdot(\hbar)^{2}}{\frac{4 \pi}{3} \cdot\left(\frac{J c^{2}}{\hbar}\right)^{3}} \equiv 0
\end{aligned}
$$

i.e. in order to obtain, in a sense a Quintic equation which can be solved [5],

$$
\begin{aligned}
& (\Delta t)^{5}-\frac{n_{\text {graviton-count }}}{\left(\frac{4 \pi T_{\text {temp }}}{d}\right)^{d-1} \cdot \frac{4 \pi}{3} \cdot\left(\frac{J c^{2}}{\hbar}\right)^{3}}(\Delta t)^{2}+\frac{16 \pi \cdot(\hbar)^{2}}{\frac{4 \pi}{3} \cdot\left(\frac{J c^{2}}{\hbar}\right)^{3}} \equiv 0 \\
& \Rightarrow A_{1}=-\frac{n_{\text {graviton-count }}}{\left(\frac{4 \pi T_{\text {temp }}}{d}\right)^{d-1} \cdot \frac{4 \pi}{3} \cdot\left(\frac{J c^{2}}{\hbar}\right)^{3}} \\
& A_{2}=\frac{16 \pi \cdot(\hbar)^{2}}{\frac{4 \pi}{3} \cdot\left(\frac{J c^{2}}{\hbar}\right)^{3}} \\
& \Rightarrow T_{\text {temp }} \text { should be negative }
\end{aligned}
$$

\section{Can We have Negative Temperature?}

This requires [14] and it is not clear that this is actually obtainable, in the experimental set up as given in our [6] input into a black hole

What else do we need?

According to the abstract of [5]:

Quote 
Let $\mathrm{a}$ and $\mathrm{b}$ be nonzero rational numbers. We show that there are an infinite number of essentially different, irreducible, solvable, quintic trinomials $\mathrm{X}^{5}+\mathrm{ax}+$ b. On the other hand, we show that there are only five essentially different, irreducible, solvable, quintic trinomials $\mathrm{x}^{5}+\mathrm{ax}^{2}+\mathrm{b}$,

namely,

$$
\begin{aligned}
& x^{5}+5 x^{2}+3, \\
& x^{5}+5 x^{2}-15, \\
& x^{5}+25 x^{2}+300, \\
& x^{5}+100 x^{2}+1000 \\
& \text { and } x^{5}+250 x^{2}+625
\end{aligned}
$$

\section{End of quote}

Aside from having a negative temperature, as for the reason given in Equation (5) we have that if [14] is satisfied and still commensurate with reference [14] that we also need to have a polynomial in delta $t$, which is commensurate with Equation (6) which is taken from the abstract in [5] and is linkable to Equation (5).

\section{Conclusion (i.e. a Necessary Condition for Quantization of Induced Kerr Newman Black Hole as Given in [6] in Terms of Reference [5] and the Last Reference [14])}

We first of all need to have a "negative" temperature. i.e. is this doable ? This has to be rigorously explored experimentally and determined.

Secondly our Equation (5) terms have to be consistently comparable to Equation (6). This requires rescaling of Equation (5) but this is doable pending dimensional analysis, and perhaps Planckian physics units.

Both these conditions would be a necessary condition for satisfying in terms of reference [5].

$(\Delta t)^{5}+A_{1} \cdot(\Delta t)^{2}+A_{2}=0$ which we state would be due our construction a necessary condition for a complete quantum gravity analysis of gravitons being emitted from a Kerr-Newman black hole.

We state that these two points have to be determined and investigated, and also that an optimal value of $\mathrm{d}$, for dimensions for a problem, involving Kerr Newman black holes would have to be ascertained in future research.

Finally, if these conditions are NOT obtained, then in line with the discussion we had in [6], we most likely have a semi classical treatment of gravity, in terms of the configuration of the Kerr Newman black hole, which is consistent with the presentations the author saw in Dice 2018, in September, 2018, which we comment upon in [6].

\section{Conflicts of Interest}

The author declares no conflicts of interest regarding the publication of this paper. 


\section{References}

[1] Liviro, M. (2005) The Equation That Couldn't Be Solved. Simon \& Schuster Paperbacks, New York.

[2] Baker, A. An Introduction to Galois Theory. http://www.maths.gla.ac.uk/ ajb/dvi-ps/Galois.pdf

[3] Higashino, T. Galois Theory: Polynomials of Degree 5 and Up. http://web.williams.edu/Mathematics/sjmiller/public_html/hudson/higashino_galoi stheory.pdf

[4] Herstein, I. (1999) Abstract Algebra. John Wiley and Sons, New York.

[5] Spearman, B. and Williams, K. (1998) On Solvable Quintics $\mathrm{X}^{5}+\mathrm{ax}+\mathrm{b}$ and $\mathrm{X}^{5}+\mathrm{ax}^{2}$ + b. Rocky Mountain Journal of Mathematics, 28.

http://people.math.carleton.ca/ williams/papers/pdf/206.pdf

[6] Beckwith, A. How a Laser Physics Induced Kerr-Newman Black Hole Can Release Gravitational Waves without Igniting the Black Hole Bomb (Explosion of a Mini Black Hole in a Laboratory).

https://www.researchgate.net/publication/327867961_HOW_A_Laser_Physics_Ind uced_KERR-NEWMAN_BLACK_HOLE_CAN_RELEASE_GRAVITATIONAL_W AVES_without_igniting_the_Black_Hole_bomb_explosion_of_a_mini_black_hole in_a_laboratory

[7] Voronin, S.M. (1975) Theorem on the Universality of the Riemann Zeta Function. Izv. Akad. Nauk SSSR, Ser. Matem., 39, 475-486. (Reprinted in Math. USSR IZv. (1975) 9, 443-445.)

[8] Ng, J. Spacetime Foam: From Entropy and Holography to Infinite Statistics and Nonlocality. https://arxiv.org/abs/0801.2962

[9] Pickett, C. and Zunda, J. (2000) Areas of the Event Horizon and Stationary Limit Surface for a Kerr Black Hole. American Journal of Physics, 68, 746-748. https://arxiv.org/abs/gr-qc/0001053 https://doi.org/10.1119/1.19536

[10] Pieres, A. (2014) AdS/CFT Correspondence in Condensed Matter. IOP Concise Physics, A. Morgan \& Claypool Publication, San Rafael.

[11] Pieres, A. AdS/CFT Correspondence in Condensed Matter. https://arxiv.org/pdf/1006.5838.pdf

[12] Davidson, E.R. (1965) On Derivations of the Uncertainty Principle. The Journal of Chemical Physics, 42, 1461. https://doi.org/10.1063/1.1696139

[13] Ringbauer, M., Biggerstaff, D.N., Broome, M.A., Fedrizzi, A., Branciard, C. and White, A.G. (2014) Experimental Joint Quantum Measurements with Minimum Uncertainty. Physical Review Letters, 112, 020401.

[14] Park, M.-I. (2007) Thermodynamics of Exotic Black Holes, Negative Temperature, and Bekenstein-Hawking Entropy. Physics Letters, B647, 472-476.

https://arxiv.org/abs/hep-th/0602114 